\title{
The impact of a community food initiative in changing the 'food culture' of a community: a focus on mothers with young children in Skelmersdale, West Lancashire
}

\author{
R. Gregg, B. Ellahi and P. Cox \\ University of Chester, Department of Biological Sciences, Parkgate Road, Chester CHI 4BJ, UK
}

The link between a nutritionally-poor diet and the development of chronic disease is well established ${ }^{(1)}$. The incidence of such disease is of particular concern amongst population groups in areas of high socio-economic deprivation ${ }^{(2)}$. Community-based initiatives (CBI) have been gaining in popularity as a method to direct health promotion efforts at low-income populations ${ }^{(3)}$. Evaluation of food and health CBI has focused in the main on intended outcomes. The opportunity to examine unintended outcomes and the mechanisms and triggers that make an intervention successful could be increasingly valued as part of the evaluation design.

The present research aims to examine the role and outcomes of a community-based food and health intervention in relation to processes of behavioural change and to broader socio-economic influences. This objective is examined from both client- and non-client-based perspectives using mothers with young children as the focus.

The methodology is informed by social research and takes a phenomenological approach using ethnographic data-recording techniques $^{(4)}$. This combined stance is taken in order to reflect both nutritional and social (community development) outcomes of the intervention. Incorporating non-clients allows the evaluation to account for the key influences, enablers and barriers for participants and, importantly for public health research, to determine how much of any change is socially driven, i.e. any 'background noise'.

Early analysis of findings relating to clients indicates the importance of individual capacity (self-efficacy) and problem-solving capabilities for engaging in healthy eating patterns, resulting from addressing a number of common barriers in this population. The comparison with non-clients has brought to light the complex and contending influences involved in food choice, and thus far has enabled an understanding of a 'hierarchy of influences' for mothers of young children (Figure).

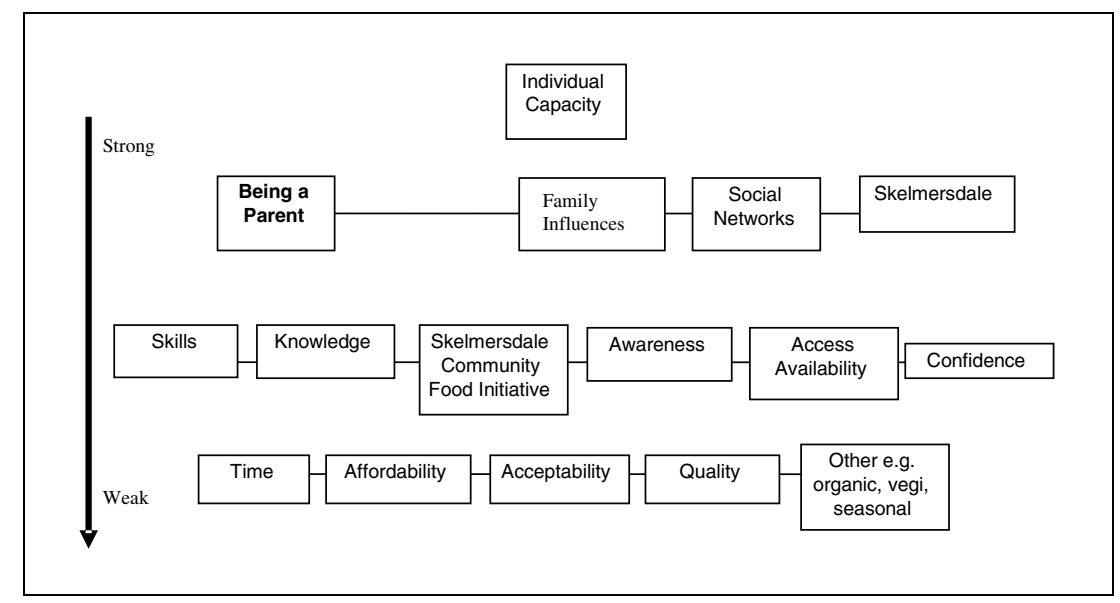

Figure. Hierarchy of influence for clients.

The present research provides an insight into the effect of a CBI on the food culture of a given population. The research has enabled an understanding of the role and impact of the intervention on the wider processes of change (successful and unsuccessful) involved in any given community setting. Further analysis will aim to develop a deeper understanding of the broader socio-economic factors that influence food and nutrition behaviour. Finally, it aims to inform methodological approaches by developing an analysis method for further evaluation of CBI.

1. World Health Organization (2003) Diet, Nutrition and the Prevention of Chronic Disease. WHO Technical Report Series no. 916. Geneva: WHO.

2. James WPT, Nelson M, Ralph A et al. (1997) Br Med J 314, 1545

3. Dowler E \& Caraher M (2003) Polit $Q \mathbf{7 4}, 57-65$

4. Bryman A (2001) Social Research Methods, 2nd ed. Oxford: Oxford University Press. 